\title{
High-dose isoniazid in the shorter-course multidrug-resistant tuberculosis regimen in the Republic of Moldova
}

\author{
To the Editor:
}

Since May 2016 the World Health Organization (WHO) has recommended the treatment of multidrug-resistant tuberculosis (MDR-TB) patients with a standardised treatment regimen of 9-12 months duration if patients fulfil specific eligibility criteria [1]. This shorter-course MDR-TB treatment regimen consists of a combination of seven drugs (clofazimine, ethambutol, high-dose isoniazid, kanamycin, moxifloxacin, prothionamide and pyrazinamide) for 4-6 months, followed by four drugs (moxifloxacin, clofazimine, pyrazinamide and ethambutol) for 5 months. It is based on the results from recent observational cohort studies performed in Bangladesh [2], Cameroon [3] and Niger [4] where this or similar treatment regimens led to high cure rates for MDR-TB. Shortly after the WHO proposed the shorter-course MDR-TB regimen, substantial concerns were raised about the applicability of this regimen for patients with MDR-TB in Europe, where many circulating strains of Mycobacterium tuberculosis have additional resistance to 2nd-line anti-tuberculosis (anti-TB) drugs. Based on results from existing databases with comprehensive records of the drug-susceptibility patterns of $M$. tuberculosis strains in patients with MDR-TB, several groups reported independently that less than $10 \%$ of MDR-TB patients from the WHO European Region were likely to be eligible for this regimen [5-8].

We read with interest the correspondence by HeLDAL et al. [9], which suggested that exclusion criteria should be interpreted more liberally to allow more patients with MDR-TB to be treated with the shorter-course regimen in Europe. We are concerned that such suggestions are being made in the absence of clinical evidence applicable to the geographic region in question and we provide further evidence here as to why this shorter-course treatment regimen should be considered with caution for patients in Europe.

One of the components of the WHO-proposed, shorter-course MDR-TB regimen is high-dose isoniazid. This recommendation is made under the assumption that treatment with $15-20 \mathrm{mg} \cdot \mathrm{kg}^{-1}$ body weight of isoniazid may be effective in M. tuberculosis strains with low-level isoniazid resistance due to mutations in the inhA promotor at positions 8,15 or 16 . There is general consensus that high-level isoniazid resistance due to a mutation in the kat $G$ gene at position 315 cannot be overcome by high-dose isoniazid treatment [10]. At the National TB Reference Laboratory of the Phthisiopneumology Institute in Chişinău, Moldova, one of the high-burden countries for MDR-TB in Eastern Europe, we evaluated all the results of molecular drug-resistance testing on $M$. tuberculosis strains performed by GenoType MTBDRplus v.2 (Hain Lifescience GmbH, Nehren, Germany) line probe assay between 2010 and 2016. This analysis then allowed us to estimate the proportion of patients with MDR-TB that might benefit from high-dose isoniazid therapy as part of the short-course regimen.

From a total of 5368 line probe assay evaluations, 4570 gave valid results (table 1). In 2638 strains, mutations were identified that suggested resistance to both isoniazid and rifampicin, this being indicative of MDR-TB. In 2323 of these 2638 strains (88.1\%), a mutation in the kat G gene at position 315 was present, suggesting high-level isoniazid resistance. Only 20 out of 2638 strains $(0.7 \%)$ had a mutation in the inhA promotor alone while in 295 out of 2638 strains (11.2\%) the location of a mutation could not be identified by the line probe assay. This data demonstrates that almost $90 \%$ of $M$. tuberculosis strains from patients with MDR-TB in the Republic of Moldova have a high-level of resistance to isoniazid.

@ERSpublications

High-dose isoniazid should not be part of a standardised treatment regimen for patients with MDRTB in Moldova http://ow.ly/zUte30eTtRW

Cite this article as: Chesov D, Ciobanu N, Lange C, et al. High-dose isoniazid in the shorter-course multidrug-resistant tuberculosis regimen in the Republic of Moldova. Eur Respir J 2017; 50: 1701340 [https://doi.org/10.1183/13993003.01340-2017]. 
TABLE 1 Isoniazid and rifampicin resistance profiles in multidrug-resistant tuberculosis (MDR-TB) strains from the Republic of Moldova, 2010-2016

\begin{tabular}{lc} 
Assay results & Valid tests \\
\hline Total valid tests $\mathbf{n}$ & 4570 \\
General resistance profile & \\
$\quad$ Isoniazid susceptible, rifampicin susceptible & $1213(26.5)$ \\
Isoniazid resistant, rifampicin susceptible & $521(11.4)$ \\
$\quad$ Isoniazid susceptible, rifampicin resistant & $198(4.3)$ \\
Isoniazid resistant, rifampicin resistant (MDR) & $2638(57.7)$ \\
Isoniazid resistance in MDR & \\
katG 315T mutation without known inhA mutations & $883(33.5)$ \\
inhA 8C, 8A, 15T or 16G mutation without known katG mutations & $20(0.7)$ \\
katG 315T mutation and inhA 8C, 8A, 15T or 16G mutation & $1440(54.6)$ \\
MDR without known katG or inhA mutations ${ }^{\#}$ & $295(11.2)$ \\
Rifampicin resistance in MDR & \\
rpoB 531L mutation & $1995(75.6)$ \\
rpoB 526Y mutation & $24(0.9)$ \\
rpoB 526D mutation & $5(0.2)$ \\
rpoB 516V mutation & $66(2.5)$ \\
Multiple rpoB mutations & $49(1.9)$ \\
MDR without known rpoB mutations ${ }^{\#}$ & $499(18.9)$
\end{tabular}

Data are presented as $n(\%)$ unless otherwise stated. Results were obtained by line probe assay using GenoType MTBDRplus v.2 (Hain Lifescience GmbH, Nehren, Germany). \#: strains defined as MDR by line probe assay results but without a signal in mutation probes while missing a signal in at least one wild-type probe.

Results from this study strongly suggest that high-dose isoniazid should not be part of a standardised treatment regimen for patients with MDR-TB in the Republic of Moldova or other parts of Europe [11]. A one-size-fits-all solution, like the WHO recommendation for the short-course MDR-TB treatment regimen, is not applicable for many patients outside of Bangladesh, Niger and Cameroon where this regimen has been highly effective. In Europe (and probably in other parts of the world) it is time to move away from standardised treatment regimens towards individualised MDR-TB therapies. For the choice of an individualised MDR-TB treatment regimen, physicians should be guided by drug availability and by the results of comprehensive molecular drug resistance testing (e.g. by line probe assay). Now that these tools are affordable even in the poorest country in Europe, we can move away from solutions based on fixed drug combinations and towards tailor-made therapies (except when all drugs in a treatment combination are novel and the likelihood that $M$. tuberculosis is resistant to any of the compounds is low).

Dumitru Chesov ${ }^{1,6}$, Nelly Ciobanu ${ }^{2,6}$, Christoph Lange ${ }^{3,4,5}$, Jan Heyckendorf $\oplus^{3}$ and Valeriu Crudu ${ }^{2}$

${ }^{1}$ Dept of Pneumology and Allergology, State University of Medicine and Pharmacy "Nicolae Testemitanu", Chișinău, Republic of Moldova. ${ }^{2}$ National TB Reference Laboratory, Phthisiopneumology Institute "Chiril Draganiuc", Chişinău, Republic of Moldova. ${ }^{3}$ Division of Clinical Infectious Diseases, German Center for Infection Research (DZIF), Research Center Borstel, Borstel, Germany. ${ }^{4}$ International Health/Infectious Diseases, University of Lübeck, Lübeck, Germany. ${ }^{5}$ Dept of Medicine, Karolinska Institute, Stockholm, Sweden. ${ }^{6}$ These authors contributed equally.

Correspondence: Dr D. Chesov, Dept of Pneumology and Allergology, State University of Medicine and Pharmacy "Nicolae Testemitanu", 165, Ştefan cel Mare şi Sfînt Bd., Chişinău, MD-2004, Republic of Moldova.

E-mail: dumitru.chesov@usmf.md

Received: July 042017 | Accepted: Aug 052017

Support statement: Christoph Lange and Jan Heyckendorf are supported by the Deutsches Zentrum für Infektionsforschung (DZIF; the German Center for Infection Research).

Conflict of interest: Disclosures can be found alongside this article at erj.ersjournals.com

\section{References}

1 Falzon D, Schünemann HJ, Harausz E, et al. World Health Organization treatment guidelines for drug-resistant tuberculosis, 2016 update. Eur Respir J 2017; 49: 1602308.

2 Van Deun A, Aung KJM, Halim MA, et al. Short, highly effective, and inexpensive standardized treatment of multidrug-resistant tuberculosis. Am J Respir Crit Care Med 2010; 182: 684-692. 
3 Kuaban C, Noeske J, Rieder HL, et al. High effectiveness of a 12-month regimen for MDR-TB patients in Cameroon. Int J Tuberc Lung Dis 2015; 19: 517-524.

4 Piubello A, Harouna SH, Souleymane MB, et al. High cure rate with standardised short-course multidrug-resistant tuberculosis treatment in Niger: no relapses. Int J Tuberc Lung Dis 2014; 18: 1188-1194.

5 Lange C, Duarte R, Frechet-Jachym M, et al. Limited benefit of the new shorter multidrug-resistant tuberculosis regimen in Europe. Am J Respir Crit Care Med 2016; 194: 1029-1031.

6 van der Werf MJ, Hollo V, Ködmön C, et al. Eligibility for shorter treatment of multidrug-resistant tuberculosis in the European Union. Eur Respir J 2017; 49: 1601992.

7 Balabanova Y, Fiebig L, Ignatyeva O, et al. Multidrug-resistant TB in Eastern region of the EU: is the shorter regimen an exception or a rule? Thorax 2017; 72: 850-852.

8 Sotgiu G, Tiberi S, D'Ambrosio L, et al. Faster for less: the new "shorter" regimen for multidrug-resistant tuberculosis. Eur Respir J 2016; 48: 1503-1507.

9 Heldal E, Van Deun A, Chiang C-Y, et al. Shorter regimens for multidrug-resistant tuberculosis should also be applicable in Europe. Eur Respir J 2017; 49: 1700228.

10 Dominguez J, Boettger EC, Cirillo D, et al. Clinical implications of molecular drug resistance testing for Mycobacterium tuberculosis: a TBNET/RESIST-TB consensus statement. Int J Tuberc Lung Dis 2016; 20: 24-42.

11 van Leth F, Guenther G, Hoffmann $\mathrm{H}$, et al. Reply from the authors of limited benefit of the new shorter MDR-TB regimen in Europe. Am J Respir Crit Care Med 2017; in press [https://doi.org/10.1164/rccm. 201705-0995LE]

\title{
A katG 315 mutation alone should not lead to exclusion of isoniazid in treatment of multidrug-resistant tuberculosis
}

\begin{abstract}
From the authors:
We thank D. Chesov and co-workers for sharing data on isoniazid-resistant strains in Moldova as a comment on our paper on shorter regimens for the treatment of multidrug-resistant tuberculosis (MDR-TB) [1]. In strains from MDR-TB patients, a high proportion (88\%) had a mutation in the katG gene at position 315, results which "...strongly suggest that high-dose isoniazid should not be part of a standardised treatment regimen for patients with MDR-TB...", based on the "...general consensus that high-level isoniazid resistance due to a mutation in the $\mathrm{katG}$ gene at position 315 cannot be overcome by high-dose isoniazid treatment...". They refer to a recent TBNET/RESIST-TB consensus statement [2] which says that molecular testing for isoniazid resistance should be done since "...it offers the possibility to add INH to a second-line drug regimen in the absence of a katG 315 mutation...”.

We do not agree with this conclusion because there are a number of studies, discussed by amongst others RIEDER et al. [3] and Отто-KNAPP et al. [4], which compare genotypic and phenotypic drug-susceptibility testing results, and which indicate that strains with this mutation have highly variable minimum inhibitory concentrations and that a majority of strains with low or moderate-level resistance can be effectively treated with isoniazid at normal or high doses. Finding this mutation should not therefore be the sole reason for exclusion of isoniazid from the treatment regimen. Indeed, a previous TBNET consensus statement recommended that dosing of isoniazid should be adjusted according to the minimum inhibitory concentration test results whenever possible [5]. Furthermore, the shortened regimen no longer counts on high-dose isoniazid as a major drug but only as a less toxic companion, since full susceptibility was not expected in the original design [6].
\end{abstract}

@ERSpublications

A katG 315 mutation alone should not lead to exclusion of isoniazid in treatment of multidrugresistant tuberculosis http://ow.ly/18cd30fiJfs

Cite this article as: Heldal E. A katG 315 mutation alone should not lead to exclusion of isoniazid in treatment of multidrug-resistant tuberculosis. Eur Respir J 2017; 50: 1701696 [https://doi.org/10.1183/ 13993003.01696-2017]. 


\section{Einar Heldal}

Norwegian Institute of Public Health, Oslo, Norway.

Correspondence: E. Heldal, Norwegian Institute of Public Health, P.O. Box 4404, Nydalen, Oslo 0403, Norway. E-mail: einar.heldal@gmail.com

Received: Aug 192017 | Accepted: Aug 212017

Conflict of interest: None declared.

\section{References}

1 Heldal E, Van Deun A, Chiang C-Y, et al. Shorter regimens for multidrug-resistant tuberculosis should also be applicable in Europe. Eur Respir J 2017; 49: 1700228.

2 Dominguez J, Boettger EC, Cirillo D, et al. Clinical implications of molecular drug resistance testing for Mycobacterium tuberculosis: a TBNET/RESIST-TB consensus statement. Int J Tuberc Lung Dis 2016; 20: 24-42.

3 Rieder HL, Van Deun A. Rationale for high-dose isoniazid in the treatment of multidrug-resistant tuberculosis. Int J Tuberc Lung Dis 2017; 21: 123-124.

4 Otto-Knapp R, Vesenbeckh S, Schönfeld $\mathrm{N}$, et al. Isoniazid minimal inhibitory concentrations of tuberculosis strains with kat $\mathrm{G}$ mutation. Int J Tuberc Lung Dis 2016; 20: 1275-1276.

5 Lange C, Abubakar I, Alffenaar JW, et al. Management of patients with multidrug-resistant/extensively drug-resistant tuberculosis in Europe: a TBNET consensus statement. Eur Respir J 2014; 44: 23-63.

6 Van Deun A, Chiang C-Y. Shortened multidrug-resistant tuberculosis regimens overcome low-level fluoroquinolone resistance. Eur Respir J 2017; 49: 1700223.

\section{Lack of evidence of isoniazid efficacy for the treatment of MDR/XDR-TB in the presence of the katG $315 \mathrm{~T}$ mutation}

To the Editor:

We are grateful for the response by E. Heldal to our correspondence. Similar to other reports [1] from the World Health Organization (WHO) Euro-Region we found that a high proportion (88\%) of Mycobacterium tuberculosis strains from patients with multidrug-resistant (MDR)/extensively drug-resistant (XDR) tuberculosis (TB) in the Republic of Moldova had a katG 315T mutation, which is strongly suggestive that high-dose isoniazid should not be part of a standardised treatment regimen for patients with MDR/XDR-TB in this country.

We respectfully disagree with the conclusion made by E. Heldal, as related to the presence of the kat $G$ 315 T mutation, that "... a majority of strains with low or moderate-level resistance can be effectively treated with isoniazid at normal or high doses..." as this statement is not sufficiently supported by the scientific literature.

In 2011 the Clinical and Laboratory Standards Institute (CLSI) defined a breakpoint for isoniazid at $0.2 \mathrm{mg} \cdot \mathrm{L}^{-1}$ for Middlebrook 7H10 media [2]. CLSI also set $1 \mathrm{mg} \cdot \mathrm{L}^{-1}$ as an additional breakpoint in order to define low-level resistance and identify patients that may possibly profit from high-dose isoniazid treatment. Full range minimum inhibitory concentration (MIC) testing in $7 \mathrm{H} 10$ showed an MIC distribution from 0.032 to $0.125 \mathrm{mg} \cdot \mathrm{L}^{-1}$ for wild-type isolates $(\mathrm{n}=79)$ [3]. In a recent cohort of patients with MDR/XDR-TB from Germany and Sweden, all 18 strains of M. tuberculosis with a mutation in katG

@ERSpublications

Further studies are needed to address the possible benefits of high-dose isoniazid therapy adequately http://ow.ly/XDcd30fiFeR

Cite this article as: Chesov D, Ciobanu N, Lange C, et al. Lack of evidence of isoniazid efficacy for the treatment of MDR/XDR-TB in the presence of the katG 315T mutation. Eur Respir J 2017; 50: 1701752 [https://doi.org/10.1183/13993003.01752-2017]. 
315T had an MIC for isoniazid of $>3 \mathrm{mg} \cdot \mathrm{L}^{-1}$ in liquid culture (MGIT) ( . Heyckendorf and co-workers, unpublished results). In 52 additional isolates with kat $G 315 \mathrm{~T}$ mutations without other known resistance mechanisms for isoniazid, MICs were found to be between 4 and $32 \mathrm{mg} \cdot \mathrm{L}^{-1}$ on Middlebrook $7 \mathrm{H} 10$ media (T. Schön and co-workers, unpublished results). In 27 of these 52 katG 315T mutated isolates the MICs for isoniazid were $8 \mathrm{mg} \cdot \mathrm{L}^{-1}$. Thus, most isolates with a $k a t G 315 \mathrm{~T}$ mutation showed MICs at least 30 times higher than current breakpoints and wild-type isolates and not a single strain out of 68 tested $M$. tuberculosis strains with isoniazid resistance due to a mutation in $k a t G 315$ had a $\mathrm{MIC}<3 \mathrm{mg} \cdot \mathrm{L}^{-1}$ by two different methods.

In the correspondence by OTTO-KNAPP et al. [4], referred to by E. Heldal as a study which indicates that strains with $k a t G 315 \mathrm{~T}$ mutations have highly variable MICs, the authors defined intermediate level drug resistance to isoniazid by an MIC of $>1$ and $<5 \mathrm{mg} \cdot \mathrm{L}^{-1}$ using Middlebrook $7 \mathrm{H} 10$ agar. The concentrations tested and the presence of other isoniazid resistance mechanisms in these isolates is not mentioned in further detail. We are unaware of an international consensus or standards on the definition of intermediate drug resistance to isoniazid within this MIC range. Furthermore, the suggestion in the same report [4] that isoniazid or any anti-tuberculosis drug is effective just if maximum serum concentrations are above the in vitro generated MIC is, to our knowledge, not supported by clinical data, nor is it consistent with current concepts of establishing clinical breakpoints [5]. The pharmacokinetic and pharmacodynamic (PK/PD) target for isoniazid needed to obtain a clinical cure has yet to be established. When considering treatment of isolates with resistance mutations and elevated MICs against isoniazid, pharmacokinetic variability should be taken into account as it strongly influences PK/PD ratios [6]. In contrast to other pathogens, clinical breakpoints including an intermediate range are not established for tuberculosis and should be based on MIC distributions, PK/PD-analysis and clinical outcome data [5].

In patients with isoniazid-monoresistant $\mathrm{TB}$ it appears that different levels of isoniazid resistance do not substantially affect TB treatment outcomes. In one study with 134 patients with culture-confirmed isoniazid-monoresistant TB, treatment success rates were similar (81.8\% versus $86.7 \%)$ between low- and high-concentration isoniazid-resistant TB [7]. In another study with 395 patients with culture-confirmed isoniazid-monoresistant pulmonary $\mathrm{TB}$, the treatment success rates were also similar in patients with high-level and low-level isoniazid-resistant $\mathrm{TB}(82.2 \%$ versus $83.4 \%)$ and among those taking anti-tuberculosis treatment with and without isoniazid (83.1\% versus $83.0 \%$ ) [8]. Of note, a cut-off of $>1 \mathrm{mg} \cdot \mathrm{L}^{-1}$ was used to define high-level isoniazid resistance in both of these trials and drug concentrations were not measured. In patients with isoniazid-resistant $\mathrm{TB}$, bacillary mutations in kat $\mathrm{G} 315 \mathrm{~T}$, but not in the inhA promotor, have been associated with unfavourable treatment outcomes [9].

The TBNET/RESIST-TB consensus statement highlights that evidence is lacking as to whether adding isoniazid to an MDR/XDR-TB regimen improves treatment outcomes when the genetic basis of the isoniazid resistance is a mutation in katG 315T [10]. Without sufficient evidence, high-dose isoniazid should not be given to patients with MDR/XDR-TB carrying strains with kat 315 mutations since the risk of toxicity may outweigh the potential benefit. Individualised therapy based on comprehensive drug susceptibility testing (DST) appears to be a more promising technique to improve therapy outcomes in MDR/XDR-TB. Early bactericidal activity studies with high-dose isoniazid in MDR/XDR-TB and clinical studies evaluating treatment outcomes in MDR/XDR-TB in patients randomised to receive high-dose isoniazid or a placebo in addition to a DST-guided regimen are needed to address the possible benefits of high-dose isoniazid therapy adequately.

Dumitru Chesov $^{1}$, Nelly Ciobanu ${ }^{2}$, Christoph Lange ${ }^{3,4,5}$, Thomas Schön ${ }^{6,7}$, Jan Heyckendorf $\odot^{3}$ and Valeriu Crudu ${ }^{2}$ ${ }^{1}$ Dept of Pneumology and Allergology, State University of Medicine and Pharmacy "Nicolae Testemitanu", Chişinău, Republic of Moldova. ${ }^{2}$ National TB Reference Laboratory, Phthisiopneumology Institute "Chiril Draganiuc", Chişinău, Republic of Moldova. ${ }^{3}$ Division of Clinical Infectious Diseases, German Center for Infection Research (DZIF), Research Center Borstel, Borstel, Germany. ${ }^{4}$ International Health/Infectious Diseases, University of Lübeck, Lübeck, Germany. ${ }^{5}$ Dept of Medicine, Karolinska Institute, Stockholm, Sweden. ${ }^{6}$ Dept of Infectious Diseases and Clinical Microbiology, Kalmar County Hospital, Kalmar, Sweden. ${ }^{7}$ Dept of Clinical and Experimental Medicine, Division of Medical Microbiology, Linköping University, Linköping, Sweden.

Correspondence: D. Chesov, Dept of Pneumology and Allergology, State University of Medicine and Pharmacy "Nicolae Testemitanu", 165, Ştefan cel Mare şi Sfînt Bd., Chişinău, MD-2004, Republic of Moldova.

E-mail: dumitru.chesov@usmf.md

Received: Aug 262017 | Accepted: Aug 292017

Conflict of interest: Disclosures can be found alongside this article at erj.ersjournals.com 


\section{References}

1 van Leth F, Guenther G, Hoffmann H, et al. Reply from the authors of limited benefit of the new shorter MDR-TB regimen in Europe. Am J Respir Crit Care Med 2017; in press [https://doi.org/10.1164/rccm. 201705-0995LE].

2 Clinical and Laboratory Standards Institute. Susceptibility testing of mycobacteria, nocardiae, and other aerobic actinomycetes; approved standard. Wayne, CLSI, 2011.

3 Schön T, Jureen P, Giske CG, et al. Evaluation of wild-type MIC distributions as a tool for determination of clinical breakpoints for Mycobacterium tuberculosis. J Antimicrob Chemother 2009; 64: 786-793.

4 Otto-Knapp R, Vesenbeckh S, Schönfeld N, et al. Isoniazid minimal inhibitory concentrations of tuberculosis strains with kat $G$ mutation. Int J Tuberc Lung Dis 2016; 20: 1275-1278.

5 Kahlmeter G. The 2014 Garrod Lecture: EUCAST-are we heading towards international agreement? J Antimicrob Chemother 2015; 70: 2427-2439.

6 Pasipanodya JG, McIlleron H, Burger A, et al. Serum drug concentrations predictive of pulmonary tuberculosis outcomes. J Infect Dis 2013; 208: 1464-1473.

7 Wang TY, Lin SM, Shie SS, et al. Clinical characteristics and treatment outcomes of patients with low- and high-concentration isoniazid-monoresistant tuberculosis. PLoS One 2014; 9: e86316.

8 Chien JY, Chen YT, Wu SG, et al. Treatment outcome of patients with isoniazid mono-resistant tuberculosis. Clin Microbiol Infect 2015; 21: 59-68.

9 Huyen MNT, Cobelens FGJ, Buu TN, et al. Epidemiology of isoniazid resistance mutations and their effect on tuberculosis treatment outcomes. Antimicrob Agents Chemother 2013; 57: 3620-3627.

10 Domínguez J, Boettger EC, Cirillo D, et al. Clinical implications of molecular drug resistance testing for Mycobacterium tuberculosis: a TBNET/RESIST-TB consensus statement. Int J Tuberc Lung Dis 2016; 20: 24-42.

Copyright (CERS 2017 